\title{
Clippers the Brain Biter: An Adolescent's Experience
}

\author{
Muhamad Azamin Anuar, Asrar Abu Bakar, Mossad Abdelhak Shaban Mohamed, Taufiq \\ Hidayat Hasan
}

Kulliyyah of Medicine, International Islamic University Malaysia, Kuantan, Pahang.

Introduction: Chronic Lymphocytic Inflammation with Pontine Perivascular Enhancement Responsive to Steroids is a rare inflammatory Central Nervous System disorder prominently affecting the brainstem. We present an adolescent's reflection on this condition complicated with Epstein-Barr virus induced CNS Lymphoma. Case report: A 16-year-old boy presented about 5 years ago with a balance problem. He was diagnosed by MRI after ongoing debate whether this is juvenile multiple sclerosis. He initially responded to methylprednisolone but developed acute deterioration requiring 8 cycles of Infliximab and Methylprednisolone. He then confirmed cerebellar lymphoma 2 years later hence commenced on chemotherapy and radiotherapy after posterior fossa decompression. He gradually losses his motor skills, left hemiparesis and spasticity. He needs tracheostomy and gastrostomy due to poor bulbar function. Now, he is fully dependent and requires chest physiotherapy and suctioning and cystostomy for urinary incontinence. He has multiple PICU admissions due to recurrent aspiration, acute cerebellar oedema and post posterior fossa decompression. He worries about family breakdown as mum is his sole carer and having regular nightmare. He changed school to meet his care demands and unsure how to adapt to new environment. Social experience makes him anxious due to lack of understanding about his condition. He is evidently having low self-esteem and confidence. Discussion: Children palliative care team has been involved since diagnosis to support him and family. He is understandably depressed and clinical psychologist input bear minimal impact. He is fully aware of the current situation and his wish to rap Eminem's songs like he used to do it before. Conclusion: Early aggressive treatment in CLIPPERS aim to prevent neuroaxonal loss. However, due to bellicose nature of the condition, the prognosis is relatively poor. Managing adolescent expectation after gradual deterioration is challenging especially, he is aware that achieving 'normality' is impossible as the treatment advancement still in limbo. 